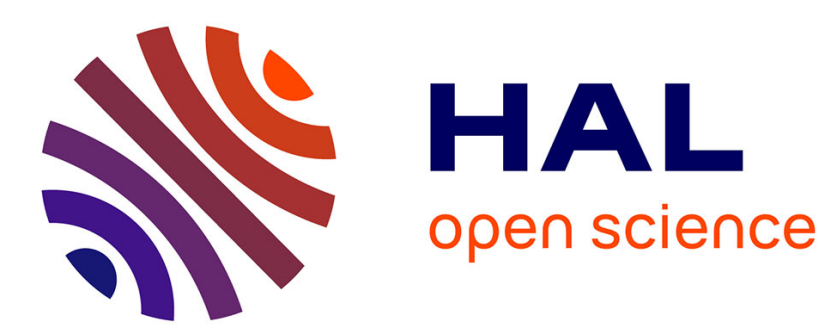

\title{
Optimized algorithm to reduce the near-field measurement time on FPGA device
}

Sebastien Serpaud, Alexandre Boyer, Sonia Ben Dhia

\section{To cite this version:}

Sebastien Serpaud, Alexandre Boyer, Sonia Ben Dhia. Optimized algorithm to reduce the near-field measurement time on FPGA device. 12th International Workshop on the Electromagnetic Compatibility of Integrated Circuits (EMC Compo 2019), Oct 2019, Haining, China. hal-02319472

\author{
HAL Id: hal-02319472 \\ https://hal.laas.fr/hal-02319472
}

Submitted on 18 Oct 2019

HAL is a multi-disciplinary open access archive for the deposit and dissemination of scientific research documents, whether they are published or not. The documents may come from teaching and research institutions in France or abroad, or from public or private research centers.
L'archive ouverte pluridisciplinaire HAL, est destinée au dépôt et à la diffusion de documents scientifiques de niveau recherche, publiés ou non, émanant des établissements d'enseignement et de recherche français ou étrangers, des laboratoires publics ou privés. 


\section{Optimized algorithm to reduce the near-field measurement time on FPGA device}

\author{
Sébastien Serpaud \\ IRT Saint-Exupéry \\ CNRS, LAAS, Univ. de Toulouse, INSA, \\ Toulouse, France \\ sebastien.serpaud@irt-saintexupery.com
}

\author{
Alexandre Boyer \\ CNRS, LAAS, Univ. de Toulouse, INSA, \\ Toulouse, France \\ alexandre.boyer@laas.fr
}

\author{
Sonia Ben Dhia \\ CNRS, LAAS, Univ. de Toulouse, INSA, \\ Toulouse, France \\ sonia.bendhia@insa-toulouse.fr
}

\begin{abstract}
This paper presents a sequential adaptive sampling algorithm in order to reduce the measurement time of near-field scan. The originality of this approach is to use a deterministic mesh swept according to a sequential progressive adaptive algorithm that defines whether a point must be captured or not. All the proposed algorithm parameters are set according to spatial field characteristics and the measurement setup. This approach is validated on the measurement of the magnetic field produced in near-field region by a FPGA device.
\end{abstract}

Keywords - EMC, NFS, Near-Field Scanning, measurement, emission, adaptive sampling

\section{INTRODUCTION}

With the current industrial economic constraints, it is necessary to evaluate electromagnetic behavior of an electronic device as early as possible in development process. If the simulation tools fail to predict effectively the electromagnetic compatibility (EMC) of complex equipment, the EMC engineer must have efficient investigation tools based on measurements. Since many years, the near-field scanning (NFS) approach applied on electronic devices is developed. The NFS performances increase industrial's interest for this EMC investigation measurement method. Limited to a robot moving a probe, the cost of NFS test bench is not expensive. The measurement is done above an electronic device. It is not strongly influenced by the set-up of equipment which ensures a high reproducibility of measurement $(<1 \mathrm{~dB})$.

Despite these qualities, the first limitation of the NFS measurement method, backed-up by the industrial, is the measurement time. In a debug context, capturing only one field component $(\mathrm{Hz})$ around a particular frequency is sufficient to investigate root cause of the EMC issue. However, in other conditions, characterizing a complex industrial electronic devices may require higher measurement time. For example, if a $200 \times 150 \mathrm{~mm}$ aeronautic calculator is scanned with $1 \mathrm{~mm}$ sampling step, if it takes 3 seconds to capture one point in the selected bandwidth of frequency $(30 \mathrm{MHz}-200 \mathrm{MHz} / 200 \mathrm{MHz}-3 \mathrm{GHz}$ with $\mathrm{RBW}=9 \mathrm{KHz} /$ $120 \mathrm{KHz}$ ), the measurement time for 2 magnetic field components is equal to 50 hours.

The orientation retained in this paper is to reduce the number of measurement points by capturing only points which bring the most information selected by a low time consuming algorithm based on the magnetic near-field characteristics. After a brief review of the principles of the sequential adaptive spatial sampling process in section II, the proposed algorithm is described in section III. In section IV and $\mathrm{V}$, validation cases are presented which highlight the performances in terms of reduction of sampling point number and measurement error compared to a full scan.

\section{SPATIAL ADAPTIVE SAMPLING AlgORITHM}

The algorithm used for this study is described in [1]. In summary, this algorithm is based on a progressive sequential meshing as defined in Fig. 1. The total mesh grid ? is built at the beginning and it is scanned progressively according to the selection criterion that defined whether or not a point must be measured. A good sample must reduce the prediction error otherwise the sample is not captured. In other words, this selection criterion defines whether or not a sample at the position pi provides relevant information about the near-field produced by the DUT noted $F$.

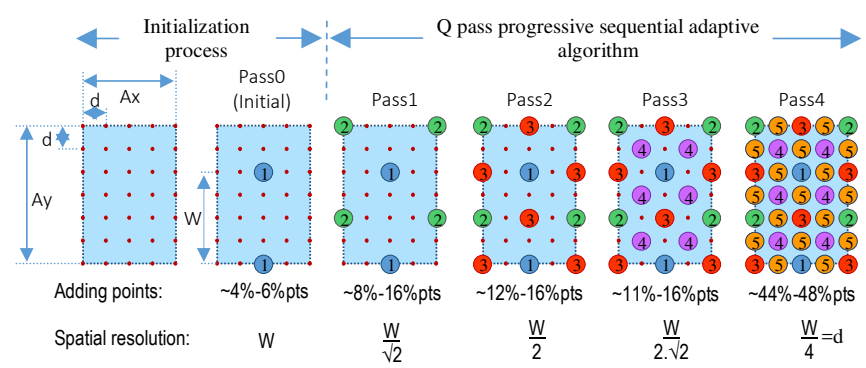

Fig. 1. Example of progressive sequential meshing where $\Omega=\{\mathrm{Ax}, \mathrm{Ay}\}$ is covered on 5 pass according to the parameters : $d$ and $W$

The first step defines the final grid resolution ' $\mathrm{d}$ ' of the regular meshing. $d$ value will be set to the radius of the magnetic probe used for the measurement.

$$
d=r_{\text {loop }}
$$

The second step is to define the first meshing used to collect the initial dataset during the "Pass 0". During this pass all points are captured. This dataset will be used to initialize the adaptive algorithm. The resolution of this initial meshing is defined by $W$ as defined by the following equation where $\mathrm{h}_{\text {meas }}$ is the height of measurement.

$$
W \leq 2 \cdot h_{\text {meas }}
$$

With $\Omega \subset \mathbb{R}^{2}$, one can bound $\Omega$ in the planar surface by $\left\{A_{x}, A_{y}\right\}$ which defines the width of $\Omega$ respectively in $\mathrm{x}$ and $\mathrm{y}$ axes. The number of points $N$ collected during the initial meshing (Pass 0 ) is defined by the following expression.

$$
N=\left\lfloor\frac{A_{x}}{W / d}+1\right\rfloor \cdot\left\lfloor\frac{A_{y}}{W / d}+1\right\rfloor
$$

A progressive spatial sweep is done on a number of sequential pass $Q$. $Q$ could be defined from $d$ and $W$ parameters according to the following equation where $\mathrm{k}$ is the biggest integer that validate $W / d \geq 2^{k}$ :

$$
Q=2 \cdot k+1
$$


On each pass, a reduced set of point $P q_{\max }=\left\{p_{q i}, q=1 \ldots Q\right\}_{i=1}^{N q_{\max }}$ is defined to progressively cover $\Omega$ at the final resolution $d$. Let $P q$ the set of $N q$ sampling points swept on each pass $q$ where $N q \leq N q_{\max }$. Note that both the evaluated number of points and spatial resolution are progressively increased pass after pass.

During the sweep of $P q_{\max }$ points, the selection criterion is built to define whether or not the value of the radiated nearfield $F$ (pqi) must be captured. The total number of collected points forms the final set $F_{M}=\left\{F\left(p_{i}\right), p i \in \Omega\right\}_{i=1}^{M}$ where $M=N+\sum_{q=1}^{Q} N_{q}$ define the total number of measurement points of $F$ at the specific positions pi. The objective is to minimize $M$, by ensuring that it allows to build an estimator that minimizes the prediction error of $F$ at any position on $\Omega$.

During iteration of $N q_{\max }$ points during the pass $q$, a selection criterion validates whether or not the value $F\left(p_{q i}\right)$ must be measured. The definition of this criterion is the main point of this algorithm. This criterion is based on the standard deviation calculated from the known values of the points $F_{v}=\left\{F\left(p_{i}\right),\right\}_{i=1}^{N_{v}}$ which neighbor $p_{q i}$. The point $p_{q i}$ is captured if $\exists p_{i} \ni F\left(p_{i}\right)>\left(\overline{F_{v}}+10 d B\right)$. To increase the effect of the closest points to $p_{q i}$, the mean value of the neighbors is weighted by the distance of each point $p_{i}$ with $p_{q i}$.

\section{CASE STUDY}

\section{A. Case study : Magnetic near-field measurement done on}

XILINX Spartan 6 FPGA device

Fig. 2 presents the test board. The frequency range starts at $5 \mathrm{MHz}$ and goes up to $1.8 \mathrm{GHz}$. Two types of magnetic field probe are used: one for the $\mathrm{Hx}$ and Hy component and another for the $\mathrm{Hz}$ component. The spatial resolution of the probes is $0.5 \mathrm{~mm}$. The signal captured by the probe is amplified by a $40 \mathrm{~dB}$ low-noise amplifier and measured by a FSP R\&S receiver. The probes are moved by a 3D scanner system at $2.5 \mathrm{~mm}$ above the top Printed Circuit Board (PCB) surface (1.1 $\mathrm{mm}$ above the top of FPGA package).

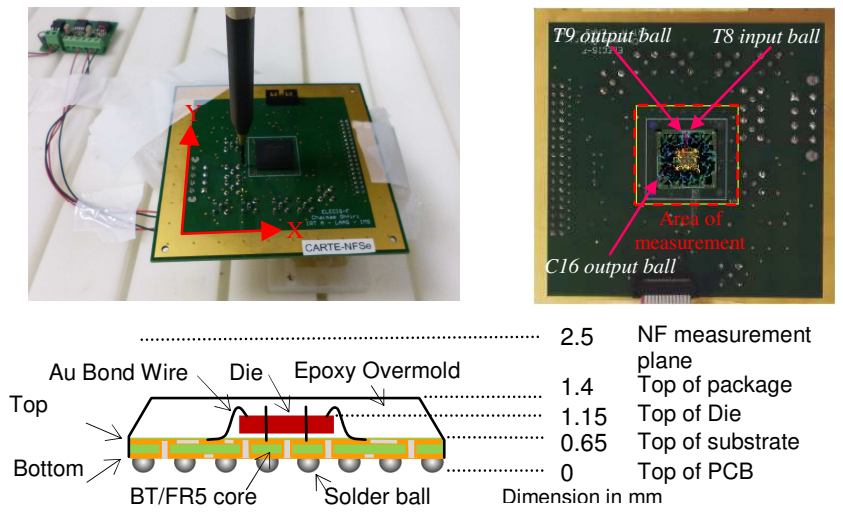

Fig. 2. FPGA

board

description

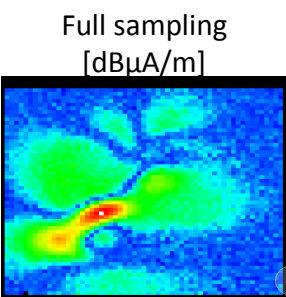

NbPts=3363pts

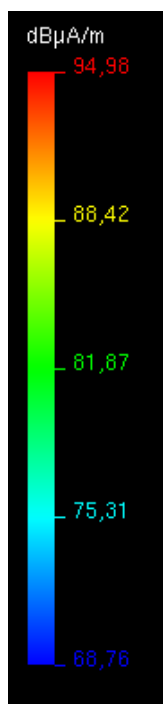

Adaptive sampling $[\mathrm{dB} \mu \mathrm{A} / \mathrm{m}]$

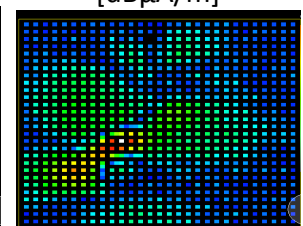

$W=1.25 \mathrm{~mm} / \mathrm{Q}=3 / \mathrm{M}=823 \mathrm{pts}$
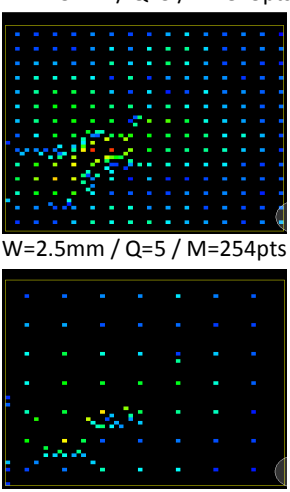

$W=5 \mathrm{~mm} / \mathrm{Q}=7 / \mathrm{M}=121 \mathrm{pts}$

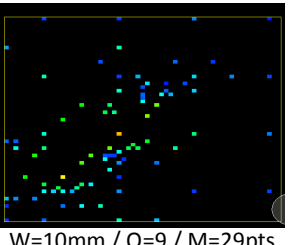

Kriging interpolation $[\mathrm{dB} \mu \mathrm{A} / \mathrm{m}]$
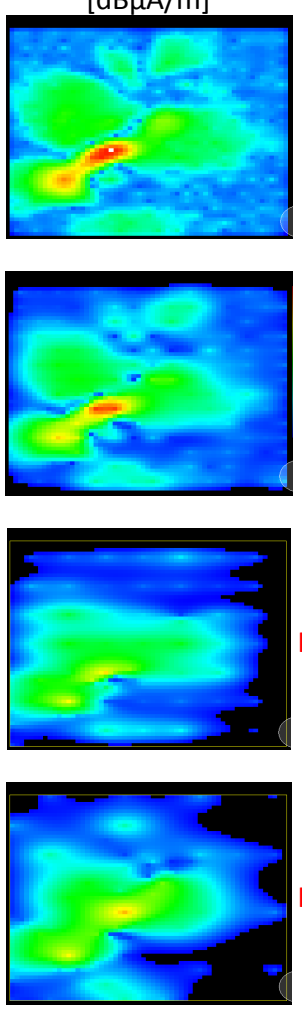

|Error $\mid$
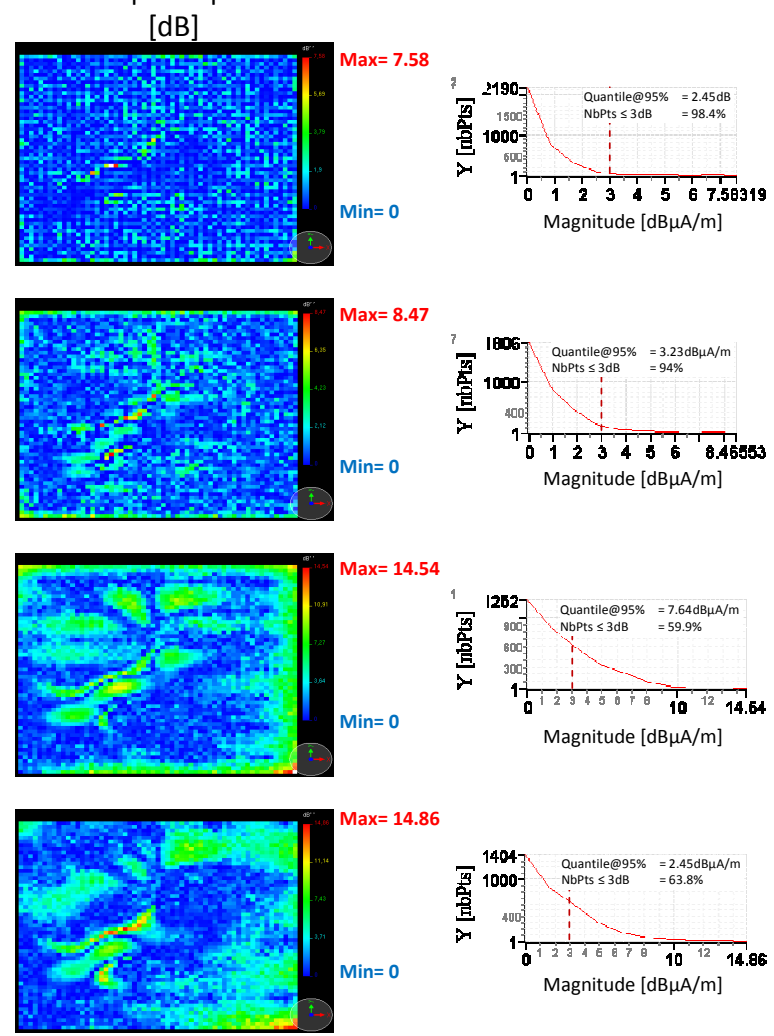

Fig. 3. Comparison between full sampling and optimized sampling from adaptive algorithm for Hy field @ 56.75MHz 


\section{VALIDATION}

The main objective of this work is to reduce the measurement time. To reach this goal, we have proposed a progressive sequential adaptive algorithm in order to reduce the number of sampling points while minimizing the error on the reconstruction of $F$ over $\Omega$. After validation of the definition of $\mathrm{W}$, we propose a discussion about these two points.

\section{A. Definition of $W$ from height of measurement}

Fig. 3 presents the comparison between full sampling measurement at $d=0.5 \mathrm{~mm}$ and optimized sampling measurement using adaptive algorithm for Hy magnetic field components process at $1.1 \mathrm{~mm}$ above the FPGA. Note for ease of understanding, all magnetic field maps are displayed for the same magnitude range for the given field component.

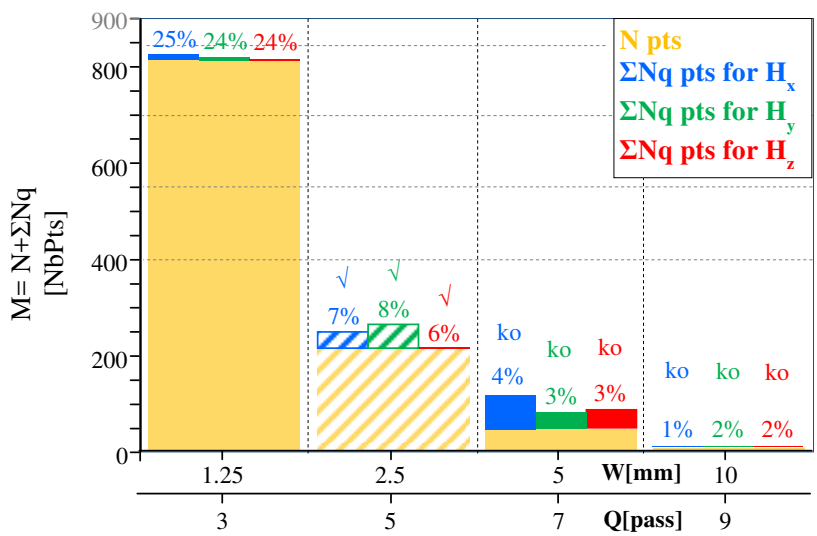

Fig. 4. Number of point captured according to W/Q parameters
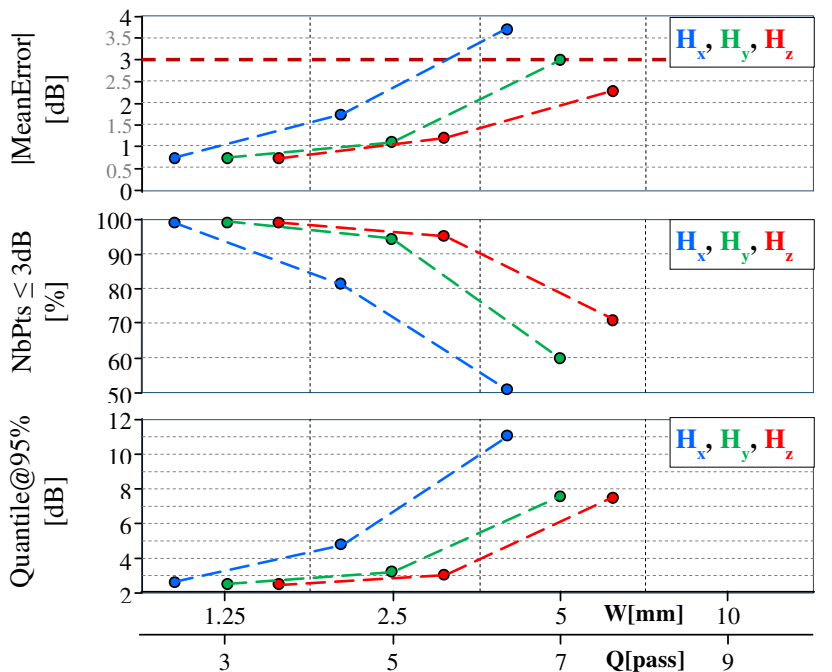

Fig. 5. Indicators about information lost on $F$ according to W/Q parameters

Fig. 4 resumes all information (about Hy but also Hx and $\mathrm{Hz}$ not completely presented in this paper) to highlight the the reduction of measurementpoints brought by the algorithm according to the $W / Q$ parameters values. The optimal configuration of $W / Q$ parameters based on the minimum number of points (without critical loss of information about $F$ ) is marked with a hatched histogram. Note the configuration is marked by 'ko' if a critical loss of information on $F$ is observed. A tick $(\sqrt{ })$ under histogram marks the theoretical optimal configuration of $W / Q$ parameters (given by the equations (2) and (4)), which is equal to 5 passes. The height of measurement is taken at $1.25 \mathrm{~mm}$. We observe a very good agreement between theoretical and optimal $\mathrm{W} / \mathrm{Q}$ parameters. This result validates the criterion on W/Q parameters defined by equations (2) and (4).

\section{B. Measurement time optimization VS lost information on $F$}

To calculate the measurement time, we assume that it takes one second to measure one point in the selected frequency range. A constant speed of $20 \mathrm{~cm} / \mathrm{s}$ is supposed to compute the probe displacement time. So for each value of $W / Q$ parameters it is possible to calculate the acquisition time related to the near-field capture over $\Omega$ ( $F_{M}$ dataset $)$, the execution time of the algorithm and near-field probe displacement time are identified.

The displacement time of the probe is small compared to the acquisition time. This is the benefit of using a regular mesh (Sukharev grid) coupling to the progressive sequential sweep[1]. The distance between two consecutive points is minimized.

In all configurations, the algorithm time is lower than one second $(\sim 50 \mathrm{~ms} \pm 10 \mathrm{~ms})$. So the most important contribution to the measurement time is the acquisition time.

In this case study, the measurement time is reduced to 19 minutes with our algorithm (using $\mathrm{W}=2.5 \mathrm{~mm} / \mathrm{Q}=5 \mathrm{pass}$ ) compared to 1 hour and 22 minutes for full scan sampling $($ gain $=10 x)$.

\section{CONCLUSION}

This paper proposes an iterative sequential adaptive sampling algorithm. This approach provides a gain of 10 on the measurement time compared to the full grid measurement, without introducing excessive measurement errors (absolute mean is $<2 \mathrm{~dB}$ ).

We have proposed a selected criterion based on the characteristic of magnetic near-field magnitude. It would be necessary to validate this algorithm on more case studies including electric near-field measurement. The selected criterion is based on real data. Multi-frequency measurements and vector measurements have not been evaluated. In this case, the definition of a specific criterion would surely be necessary. The proposed adaptive algorithm is developed for the near-field emission measurement. But it could be easily extended to near-field measurement in immunity.

\section{ACKNOWLEDGMENT}

This results have been obtained in the framework of the IRT Saint Exupery's research project FELINE. We acknowledge the financial and in-kind support (background knowledge and services) from the IRT Saint Exupery's industrial and academic members and the financial support of the French National Research Agency.

\section{REFERENCES}

[1] S. Serpaud, A Boyer, S. Ben Dhia, "Sequential adaptive sampling algorithm to reduce the near-field measurement time" EMC-Europe 2019, Barcelona, Span, September 2-6, 2019

[2] The Scikit library https://scikit-learn.org 